\title{
Effects of particulate debris on macrophage-
dependent fibroblast stimulation in coculture
}

\author{
M. Lind, M. C. D. Trindade, B. Yaszay, S. B. Goodman, R. L. Smith \\ From Stanford University School of Medicine, Stanford, USA
}

$\mathbf{T}$ he interactions between the different cell types in periprosthetic tissue are still unclear. We used a non-contact coculture model to investigate the effects of polymethylmethacrylate (PMMA) particles and human macrophage-derived soluble mediators on fibroblast activation. Macrophages were either exposed or not exposed to phagocytosable PMMA particles, but fibroblasts were not. Increasing numbers of macrophages were tested in cocultures in which the fibroblast cell number was held constant and cultures of macrophages alone were used for comparison of cytokine release. We used the release of interleukin-1 beta (IL-1 $\beta$ ), interleukin 6 (IL-6), tumour necrosis factor alpha (TNF- $\alpha$ ), lysosomal enzyme and metalloproteinase activity to assess the cultivation of macrophages and fibroblasts.

In cocultures, IL-6 release was increased 100-fold for both unchallenged and particle-challenged cultures when compared with macrophage cultures alone. Furthermore, particle-challenged cocultures had threefold higher IL-6 levels than unchallenged cocultures. Release of TNF- $\alpha$ was similar in cocultures and in macrophage cultures. $I L-1 \beta$ release in cocultures was independent of the macrophage-fibroblast ratio. Lysosomal enzyme activity and metalloproteinase activity were increased in cocultures.

Our data show that macrophages and fibroblasts in coculture significantly increase the release of IL-6 and to a less degree other inflammatory mediators; particle exposure accentuates this effect. This suggests that macrophage accumulation in fibrous tissue may

M. Lind, MD, PhD, Postdoctoral Fellow

Orthopaedic Research Laboratory, Aarhus University Hospital, Nørrebrogade, 8000 Aarhus C, Denmark.

M. C. D. Trindade, BA, Graduate Student Research Assistant

B. Yaszay, BA, Stanford Medical Student III

S. B. Goodman, MD, Associate Professor

R. L. Smith, PhD, Associate Professor

Orthopaedic Research Laboratory, Stanford University School of Medicine, 300 Pasteur Drive, R144, Stanford, California 94305, USA.

Correspondence should be sent to Dr R. L. Smith.

(C)1998 British Editorial Society of Bone and Joint Surgery 0301-620X/98/58710\$2.00 lead to elevated IL-6 levels that are much higher than those caused by particle activation of macrophages alone. This macrophage-fibroblast interaction represents a novel concept for the initiation and maintenance of the inflammatory process in periprosthetic membranes.

J Bone Joint Surg [Br] 1998;80-B:924-30.

Received 6 January 1998; Accepted after revision 15 May 1998

Wear of materials from joint prostheses produces particulate debris which induces a chronic inflammatory response in the periprosthetic tissues. This reaction involves macrophage activation and results in the formation of a periprosthetic membrane and accelerated bone resorption. ${ }^{1,2}$ The periprosthetic membrane typically consists of macrophages in a rich fibroblastic stroma. ${ }^{3,4}$ When macrophages phagocytose wear particles they become activated and secrete numerous inflammatory mediators ${ }^{5-7}$ which may interact with fibroblasts and other cells in the membrane to potentiate a chronic inflammatory reaction.

Studies in vitro have shown that wear particles activate macrophages to secrete protein and express mRNA of the proinflammatory cytokines, interleukin-1 $\beta$ (IL-1 $\beta$ ), interleukin 6 (IL-6), tumour necrosis factor alpha (TNF- $\alpha$ ), and chemokines such as macrophage-activating and chemotactic protein 1 (MCP-1) and macrophage inflammatory protein (MIP). ${ }^{8-12}$ Some of these cytokines stimulate bone resorption in vitro and may be a mechanism by which particle-induced macrophage activation contributes to the failure of endoprosthetic implants. ${ }^{13}$

Fibroblasts in culture respond to orthopaedic wear debris by a proliferative response to polymethylmethacrylate (PMMA) and metal particles including cobalt-chrome and titanium alloy. ${ }^{14-16}$ Typically, low particle doses $(<0.05 \%)$ increase fibroblast proliferation whereas higher doses decrease it. Secretion of different cytokines and growth factors from fibroblasts is also modulated by particles. ${ }^{16}$ Fibroblasts have a characteristic basal secretion of IL-6 that is increased in response to high concentrations of particles $(>0.05 \%)$ whereas low concentrations tend to increase secretion of basic fibroblast growth factor (bFGF).

In the peri-implant tissue there are complex interactions between macrophages, fibroblasts and osteoblasts. Cocul- 
ture of macrophages and osteoblasts has been shown to elevate the basal secretion of proinflammatory mediators such as PGE 2 and IL-6 in the absence of particles, and particle activation further increased secretion of mediators. ${ }^{8}$ Two further studies have investigated interactions between macrophages and fibroblasts in vitro by adding macrophage-conditioned medium to human dermal fibroblasts. ${ }^{9,17}$ Conditioned medium from macrophages activated with phagocytosable titanium alloy and polyethylene particles increased fibroblast proliferation.

Our aim was to test the hypothesis that the accumulation of macrophages stimulates fibroblasts by the release of soluble mediators, and that phagocytosable particles accentuate this process. We determined the effects of macrophages cultured with and without PMMA particles on fibroblasts in coculture using tissue-culture inserts to maintain the separation of the two cell types. Cytokine release, lysosomal enzyme activity and matrix metalloproteinase (MMP) release were quantified to assess cell activation. We used control cultures with macrophages alone to compare the response in cytokine secretion profiles in the absence of fibroblasts.

\section{Materials and Methods}

Human monocytes/macrophages. These were isolated from buffy coats obtained from healthy volunteers by Ficoll gradient centrifugation. The isolated monocytes/macrophages were cultured in RPMI 1640 medium (Gibco, Gaithersburg, Maryland) supplemented with 5\% human serum (Sigma, St Louis, Missouri) and gentamicin $(25 \mu \mathrm{g} /$ $\mathrm{ml}$ ) and allowed to adhere for 24 hours at $37^{\circ} \mathrm{C}$ with $5 \%$ $\mathrm{CO}_{2}$. Adherent monocyte cells, now designated macrophages, were released using $0.05 \%$ trypsin and $0.25 \%$ EDTA and then replated either on to $25 \mathrm{~mm}$ inserts for coculture with fibroblasts or on to 24-well plates. Macrophages were plated at different concentrations $\left(5 \times 10^{3}\right.$, $5 \times 10^{4}, 5 \times 10^{5}$ and $5 \times 10^{6}$ ) per insert and at half those densities per well in the 24-well plates. After 72 hours in RPMI 1640 medium supplemented with 5\% human serum, the cells were washed four times with phosphate-buffered saline (PBS). Macrophages in the six-well plates were cultured in $4 \mathrm{ml}$ of serum-free RPMI 1640 in each well and in the 24-well plates with $1.0 \mathrm{ml}$ of medium per well. Data are based on six separate experiments with macrophages from three different individuals. Macrophages used in coculture and alone were derived from identical buffy coats, and were plated at identical cell densities to allow comparison of release of cytokines.

Human fibroblasts. Human foreskin fibroblasts (ATCC CCD-1059SK) were cultured on $100 \mathrm{~mm}$ plates in alpha minimal essential medium ( $\alpha \mathrm{MEM})$ (Gibco, Gaithersburg, Maryland) supplemented with $10 \%$ fetal calf serum (FCS) and gentamicin $(25 \mu \mathrm{g} / \mathrm{ml})$ and allowed to adhere and proliferate for 72 hours at $37^{\circ} \mathrm{C}$ with $5 \% \mathrm{CO}_{2}$. Cells were released by using $0.25 \%$ trypsin and $0.03 \%$ EDTA, replated on to six wells at a density of 100000 cells per well, and allowed to adhere and proliferate in $\alpha$ MEM with $10 \%$ FCS for 72 hours. They were maintained in serum-free $\alpha \mathrm{MEM}$ for 24 hours before coculture with human macrophages. At this point 210000 (SD 90000 ) cells were present in each well.

Particles. The particles used for the activation of macrophages were commercially available PMMA particles, 1 to $10 \mu \mathrm{m}$ in size, with a mean diameter of $4.5 \mu \mathrm{m}$ (Polysciences, Warrington, Pennsylvania). They were sterilised overnight in $70 \%$ ethanol, and extensively washed in sterile PBS before usage in macrophage cultures.

Study design and coculture. Macrophages and fibroblasts were cocultured using the tissue-culture insert system (Nunc, Roskilde, Denmark). With this system, different cells can be cultured together in two layers with humeral contact but without direct cell contact. Fibroblasts were cultured in six-well plates and macrophages on $25 \mathrm{~mm}$ inserts with polycarbonate membranes where the humeral contact was achieved through $0.2 \mu \mathrm{m}$ pores. In this experiment macrophages were plated at different concentrations $\left(5 \times 10^{3}, 5 \times 10^{4}, 5 \times 10^{5}\right.$ and $\left.5 \times 10^{6}\right)$ per insert. At the time of the experiment $20 \%$ of the cells were adherent. The fibroblasts were plated at a density of $1 \times 10^{5}$ and proliferated to a density of $2.0 \times 10^{5}$. When expressed as a macrophage/fibroblast ratio (MFR), the different macrophage densities corresponded to $0.005,0.05,0.5$ and 5.0 MFR. We performed the cocultures with and without $0.075 \%$ PMMA particles added to the macrophages. As a control for the macrophage response to particles in the absence of fibroblasts, macrophages were cultured in 24-well plates at similar cell densities as used in the coculture experiment. They were cultured with and without $0.075 \%$ PMMA particles.

Macrophage activation. IL- $1 \beta$, IL- 6 and TNF- $\alpha$ levels were determined in conditioned medium samples from the coculture and macrophage cultures by a standard enzymelinked immunosorbent assay (ELISA) technique. All cytokines were measured by using paired antibodies from R \& D Systems (Minneapolis, Minnesota). Macrophages were examined microscopically for their morphological response to particles and phagocytosis was assessed.

Lysosomal enzyme activity. We evaluated lysosomal enzyme activity by determining hexosaminidase activity in conditioned medium. We incubated $20 \mu \mathrm{l}$ of medium with $100 \mu \mathrm{l}$ of a solution of $0.5 \mathrm{mM}$ p-nitrophenyl $\mathrm{N}$-acetyl- $\beta$-dglucosaminide and $0.05 \mathrm{M}$ sodium citrate $(\mathrm{pH} 5.0)$ in 96-well plates. The mixture was incubated for two hours at $37^{\circ} \mathrm{C}$. The enzyme reaction was stopped with the addition of $50 \mu \mathrm{l}$ of $0.5 \mathrm{M}$ sodium hydroxide. The resulting colorimetric product was measured on an ELISA reader at $405 \mathrm{~nm}$ with background correction of $595 \mathrm{~nm}$. The data are expressed as arbitrary units using a standard curve generated from serial dilutions of the enzyme $\beta-\mathrm{N}$-acetylglucosaminidase.

Matrix metalloproteinase (MMP) activity. We performed sodium dodecyl sulphate (SDS) substrate gel analysis to 
assess neutral MMP activity in samples of conditioned medium. Endogenously activated and total enzyme activity was determined by analysis of the samples with and without 4-aminophenylmercuric acetate (APMA) activation (incubation in $1.0 \mathrm{mM}$ APMA for one hour at $37^{\circ} \mathrm{C}$ ). Aliquots were electrophoresed in $10 \%$ SDS-polyacrylamide gels impregnated with $1 \mathrm{mg} / \mathrm{ml}$ of gelatin. SDS was removed by shaking in $2.5 \%$ Triton X-100 for 30 minutes at $25^{\circ} \mathrm{C}$ and the gels were subsequently incubated for 16 hours in substrate buffer $(50 \mathrm{mM}$ Tris $\mathrm{HCl}$ buffer, $\mathrm{pH} 8.0$, $10 \mathrm{mM} \mathrm{CaCl}{ }_{2}$ and $0.02 \%$ azide) at $37^{\circ} \mathrm{C}$. They were then stained with $0.5 \%$ Coomassie Brilliant Blue R 250 in $30 \%$ isopropyl alcohol and $10 \%$ acetic acid to visualise gelatinolytic activity.

Statistical analysis. Data were based on six separate experiments with macrophages from three different individuals. Macrophages used in coculture and alone were derived from identical buffy coats and were plated at identical cell densities to allow comparison of cytokine release. All data are presented as mean \pm SEM. KruskalWallis non-parametric ANOVA and Dunn tests for multiple comparisons were used to compare differences between control and stimulation groups in dose-response studies and to compare differences between cocultures and macrophage cultures. A p value <0.05 was considered significant.

\section{Results}

IL-6 release. In macrophage/fibroblast cocultures, IL-6 release ranged from 50 to $75 \mathrm{pg} / \mathrm{ml}$ in the presence or absence of particles added to the macrophages up to a macrophage/fibroblast ratio (MFR) of 0.05 (Fig. 1a). In the absence of particles, the release of IL-6 shifted abruptly when the MFR reached 0.5 and 5.0, with IL-6 levels reaching 195 and $620 \mathrm{pg} / \mathrm{ml}$, respectively (Fig. 1a). With the addition of particles to the macrophages in coculture,

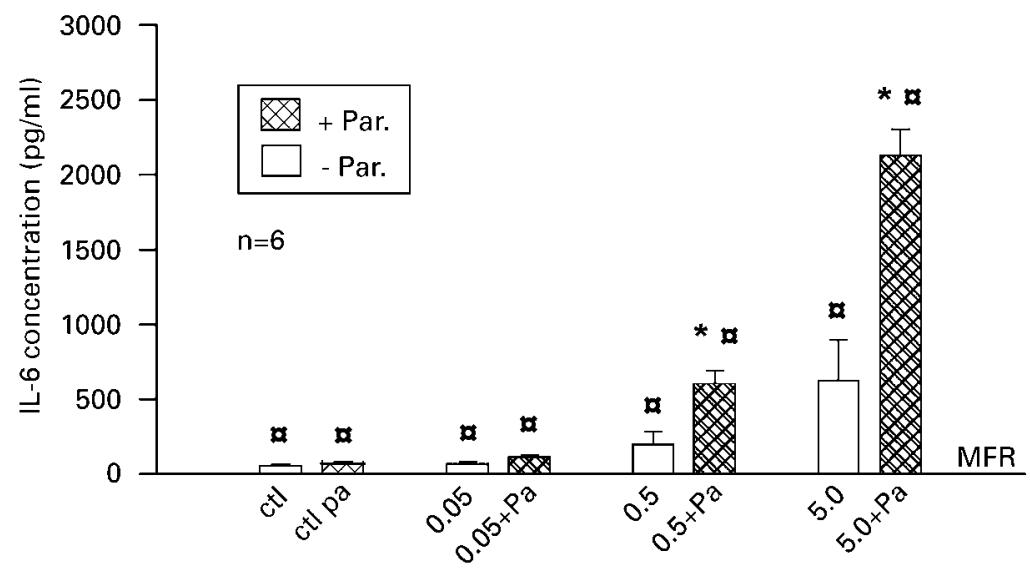

Fig. 1a

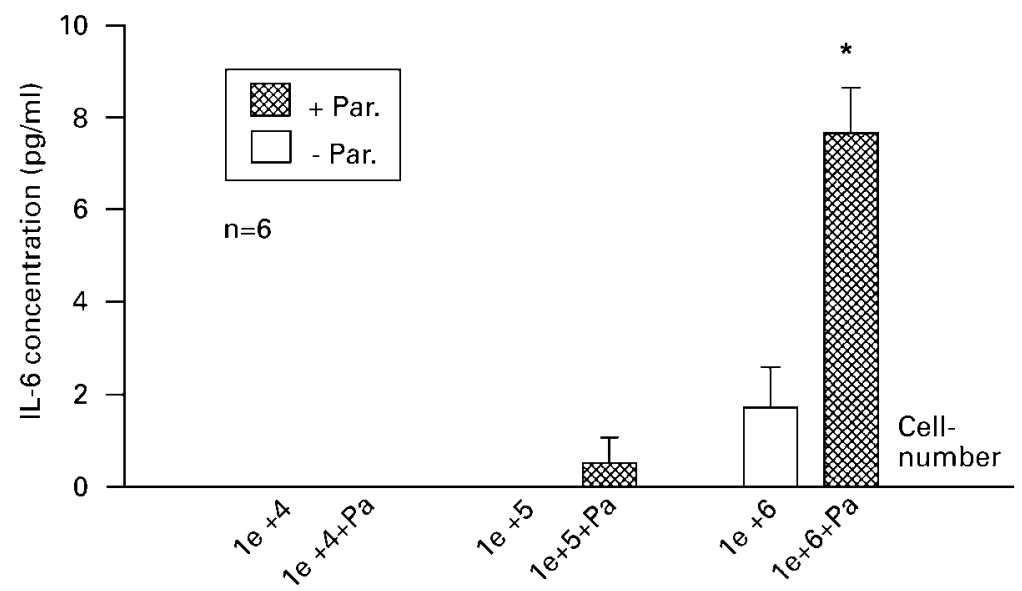

Fig. $1 b$

Figure 1a - IL-6 release in cocultures with different macrophage to fibroblast ratios (MFR) (ctl indicates fibroblast alone; $\mathrm{Pa}$ indicates particle challenge). Figure $1 \mathrm{~b}-$ IL-6 release in macrophage cultures with different cell numbers. Open bar illustrates release in cultures in which the macrophages were unchallenged by PMMA particles. Crosshatched bars illustrate release in particle-challenged cultures $(*$ indicates significant difference between unchallenged and particle-challenged cultures $(\mathrm{p}<0.05)$; $\not \alpha$ indicates significant difference between macrophage culture and coculture $(\mathrm{p}<0.05)$; $\mathrm{Pa}$ indicates particle challenge). 


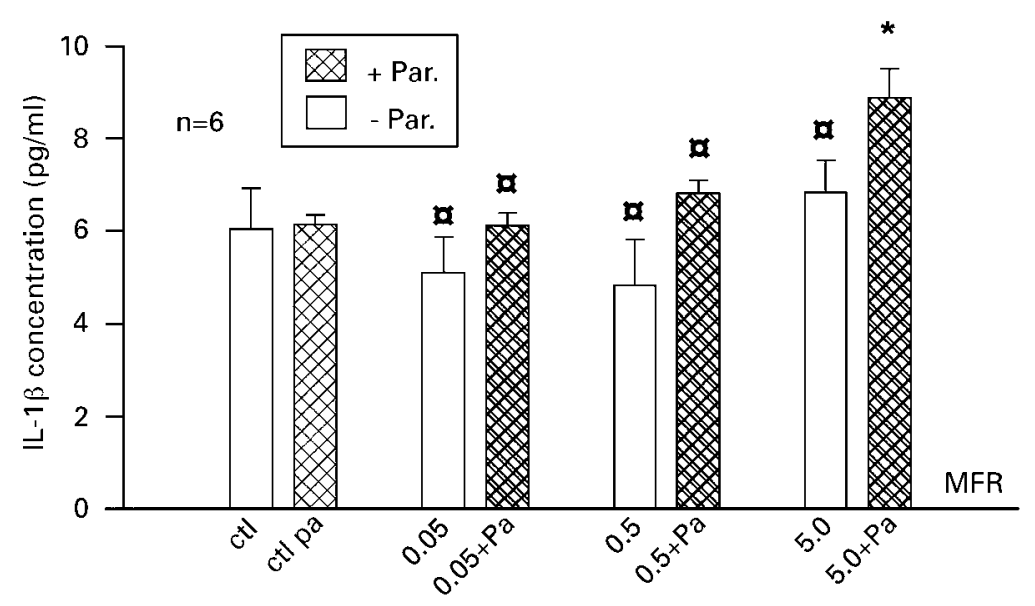

Fig. 2a

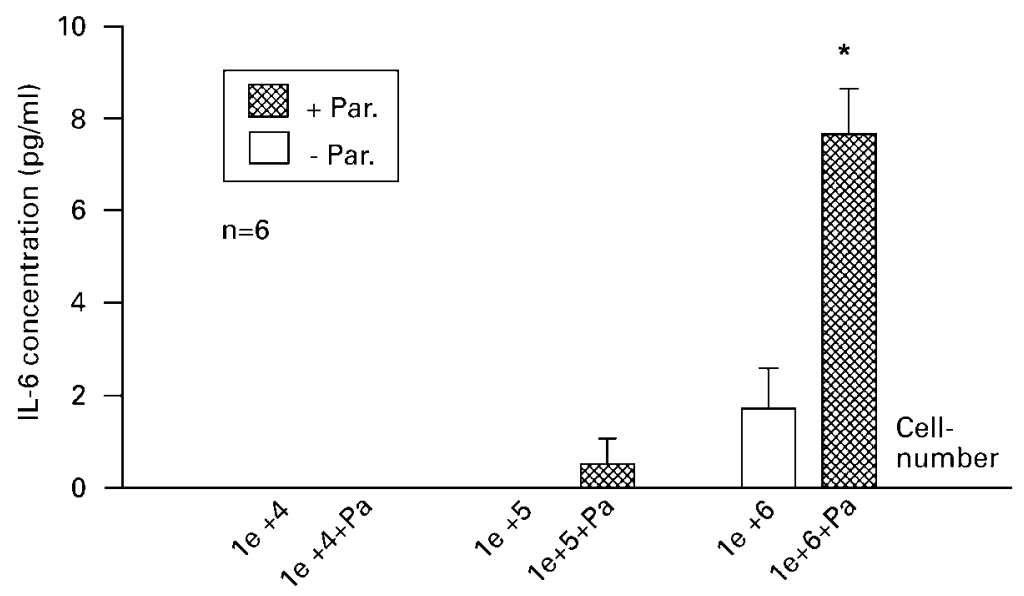

Fig. $2 b$

Figure $2 \mathrm{a}-\mathrm{IL}-1 \beta$ release in cocultures with different macrophage to fibroblast ratios (MFR) (ctl indicates fibroblasts alone; Pa indicates particle challenge). Figure $2 \mathrm{~b}-\mathrm{IL}-1 \beta$ release in macrophage cultures with different cell numbers. Open bars illustrate release in cultures in which the macrophages were unchallenged by PMMA particles. Crosshatched bars illustrate release in particle-challenged cultures (* indicates significant difference between unchallenged and particle-challenged cultures $(\mathrm{p}<0.05) ; \quad$ indicates significan difference between macrophage culture and coculture $(\mathrm{p}<0.05)$; $\mathrm{Pa}$ indicates particle challenge).

IL-6 release increased a further threefold to 600 and $2100 \mathrm{pg} / \mathrm{ml}$ for an MFR of 0.5 and 5, respectively (Fig. 1a). In macrophage cultures IL-6 was only detected at the highest cell density of unchallenged macrophages. For particle-challenged macrophages, IL-6 release occurred at a relatively low level with the two highest cell densities but increased twofold when compared with unchallenged macrophages (Fig. 1b). Tests of an MFR of 0.005 in cocultures and a cell number of $1 \times 10^{3}$ in macrophage cultures showed no response (data not shown in figures).

IL-1 $\beta$ release. In macrophage/fibroblast cocultures almost constant levels of IL- $1 \beta$ were present for all MFRs without particle challenge. For particle-challenged macrophages in coculture only an MFR of 5.0 showed increased release compared with fibroblasts alone and an MFR of 0.005 to 0.05 ( $\mathrm{p}<0.05$ ) (Fig. 2a). In macrophage cultures IL-1 $\beta$ was almost undetected in unchallenged macrophages. With particle-challenged macrophages, increased release of IL-1 $\beta$ occurred for $1 \times 10^{4}$ to $1 \times 10^{6}$ cell densities (Fig. $2 b$ ).

TNF $-\alpha$ release. In the macrophage/fibroblast cocultures, an MFR-dependent increase in the release of TNF- $\alpha$ was seen with the highest TNF- $\alpha$ concentration of $80 \mathrm{pg} / \mathrm{ml}$ present in particle-challenged macrophages at an MFR of 5.0 (Fig. 3a). In macrophage cultures, release of TNF- $\alpha$ was similar with low levels in unchallenged macrophages. Particlechallenged macrophages showed increased release of TNF$\alpha$ at the $1 \times 10^{5}$ to $1 \times 10^{6}$ cell densities with the highest concentration of TNF- $\alpha$ of $65 \mathrm{pg} / \mathrm{ml}$ occurring at a cell density of $1 \times 10^{6}$ (Fig. 3b).

Hexosaminidase activity. Detectable hexosaminidase activity was only seen at $1 \times 10^{6}$ and an MFR of 5.0 for macrophage cultures and cocultures, respectively. Particle challenge increased hexosaminidase activity approximately twofold in macrophage cultures $(p<0.05)$ whereas no difference was seen between unchallenged and particlechallenged cocultures (Fig. 4). 


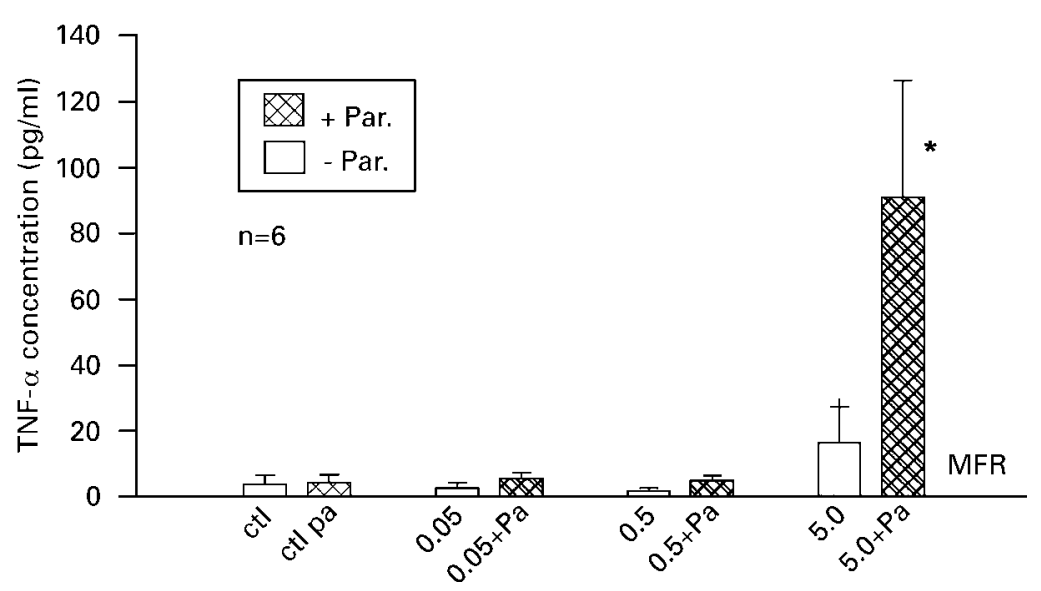

Fig. 3a

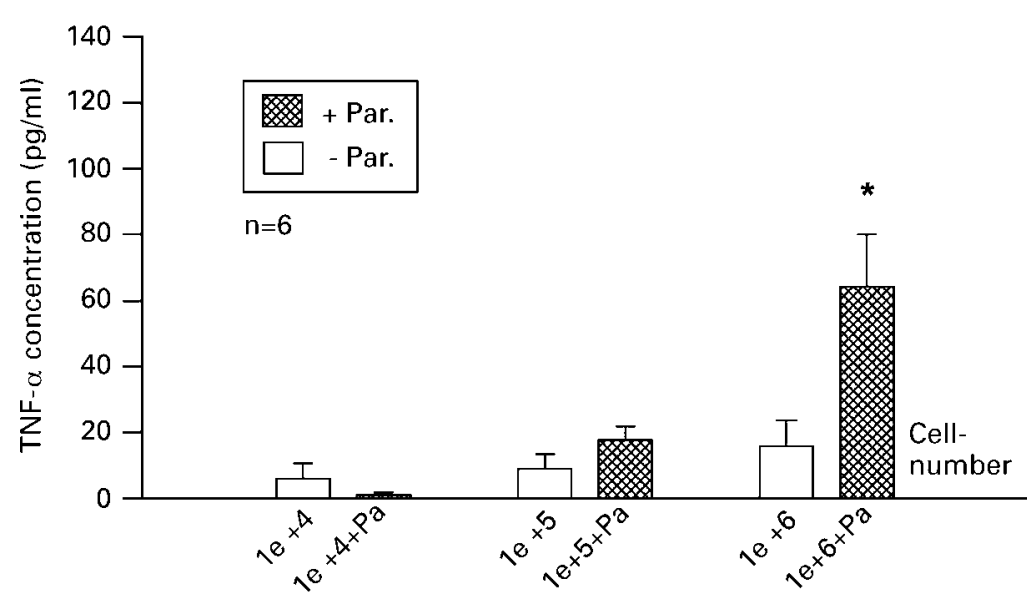

Fig. $3 b$

Figure $3 a-T N F-\alpha$ release in cocultures with different macrophage to fibroblast ratios (MFR) (ctl indicates fibroblasts alone; Pa indicates particle challenge). Figure $3 b-\mathrm{TNF}-\alpha$ release in macrophage cultures with different cell numbers. Open bars illustrate release in cultures in which the macrophages were unchallenged by PMMA particles. Crosshatched bars illustrate release in particle-challenged cultures (* indicates significant difference between unchallenged and particle-challenged cultures $(\mathrm{p}<0.05)$; $\mathrm{Pa}$ indicates particle challenge).

Matrix metalloproteinase activity. Zymographic analysis of the non-APMA-treated medium samples confirmed that MMP-9 was the predominant metalloproteinase expressed in the macrophage cultures. Addition of particles increased the MMP-9 expression and MMP-2 was just detected. In the fibroblast cultures, MMP-2 was the predominant metalloproteinase expressed in the non-APMA-treated medium samples. In the cocultures, the relative proportion of MMP-9 and MMP-2 expression was consistent with the cellular origin. They showed increased levels of the interstitial collagenase, MMP-1, however, and the addition of particles to the macrophages increased the detection of MMP-1. After APMA activation the zymographic profiles agreed with these data.

\section{Discussion}

Our study has shown for the first time that increasing the number of macrophages relative to the number of fibro- blasts in a coculture system affected the release of proinflammatory mediators. There was a substantial humeral interaction between the two cell types indicated by release of IL-6. The release of IL-1 $\beta$ and lysosomal enzyme activity from unchallenged macrophages in coculture with fibroblasts reached the same level as that of particlechallenged cultures of macrophages alone. In addition, coculture showed a particle-dependent increase in MMP-1. TNF- $\alpha$ was not increased by macrophage/fibroblast coculture. Our data suggest that a specific interaction may occur between macrophages and fibroblasts which is mediated by soluble factors.

The selective increase in the release of IL- 6 as a result of coculture may be derived from macrophages or fibroblasts or both. Both cell types are able to secrete substantial amounts of IL-6; high levels of stimulation can be obtained by lipopolysaccharide (LPS) for macrophages and IL-1 $\beta$ for fibroblasts. ${ }^{18}$ IL-1 $\beta$ may have been the stimulating mediator in our coculture model. Our data, however, did 


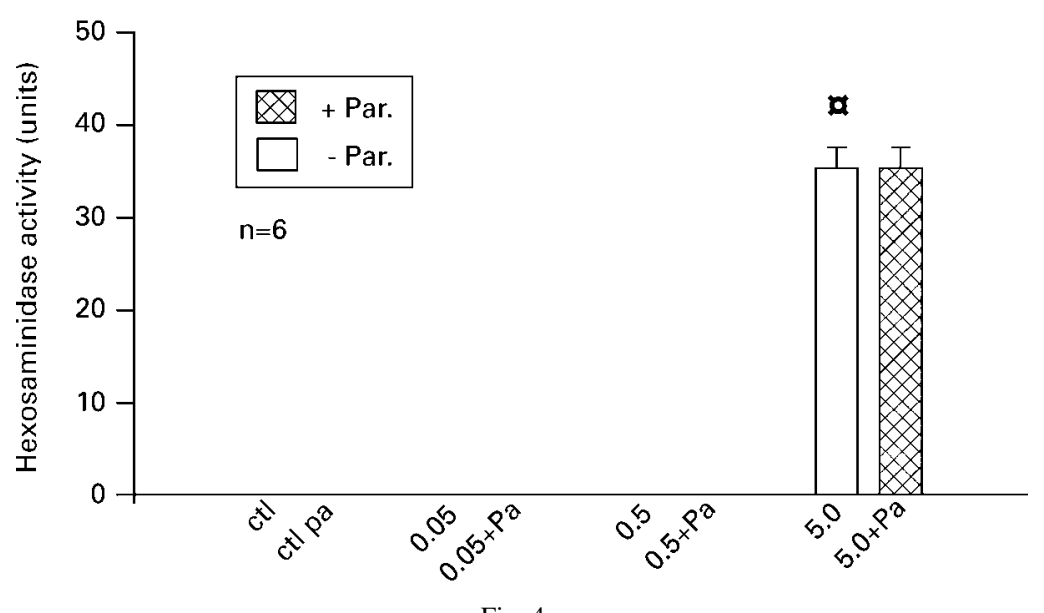

Fig. 4a

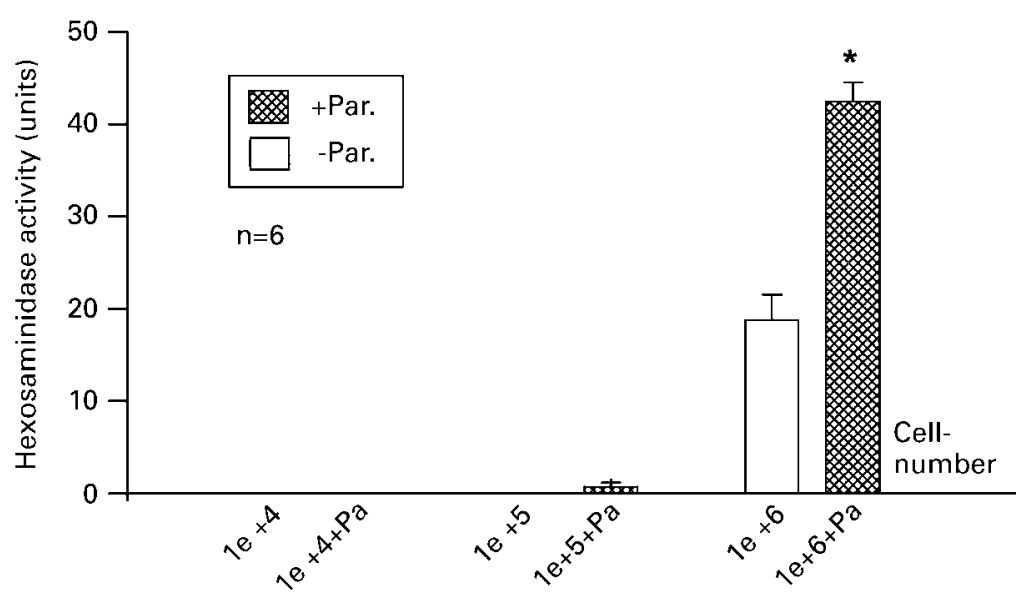

Fig. $4 b$

Figure $4 \mathrm{a}-$ Hexosaminidase release in cocultures with different macrophage to fibroblast ratios (MFR) (ctl indicates fibroblasts alone; $\mathrm{Pa}$ indicates particle challenge). Figure $4 \mathrm{~b}-$ Hexosaminidase release in macrophage cultures with different cell numbers. Open bars illustrate release in cultures in which the macrophages were unchallenged by PMMA particles. Crosshatched bars illustrate release in particle-challenged cultures $(*$ indicates significant difference between unchallenged and particle-challenged cultures $(p<0.05)$. $\mathrm{a}$ indicates significant difference between macrophage culture and coculture $(\mathrm{p}<0.05) ; \mathrm{Pa}$ indicates particle challenge).

not show increased levels of IL- $1 \beta$ in coculture with an MFR of 0.5 and 5.0. This suggests that other soluble mediators may be responsible for the increase in IL-6. One mediator that is expressed and secreted at a constant high level by macrophages is macrophage migration inhibitory factor (MIF). ${ }^{19}$ Such factors may act alone, by interactions with other mediators, or through escalation of receptors to stimulate fibroblasts to increased release of IL- $6 .{ }^{18,20,21}$

The increase in IL- 6 during interaction of macrophages and fibroblasts may be important in periprosthetic membranes in vivo, in which the high IL-6 level may contribute to accelerated bone resorption due to the stimulation of osteoclasts. $^{22,23}$ This interaction may be more important to inflammation and subsequent bone resorption in periprosthetic tissue than the cytokine release occurring from macrophages after phagocytosis and activation by wear particles.

A previous study has shown a similar humeral interaction between macrophages and osteoblasts. ${ }^{8}$ Release of
IL-6 and $\mathrm{PGE}_{2}$ was synergistically increased when macrophages and osteoblasts were cocultured when compared with isolated cultures. Addition of polyethylene particles further increased the release of these mediators. Two previous studies have shown a humeral interaction between macrophages and fibroblasts. ${ }^{9,15}$ Conditioned medium from macrophages exposed to phagocytosable particles induced a proliferative response in fibroblasts. Macrophage-fibroblast interactions involving cytokine release have been described previously. Blue et $\mathrm{al}^{24}$ showed an increase in adhesion molecules and IL- 6 and TNF- $\alpha$ when macrophages and synoviocytes were cocultured with humeral contact only. Another study showed an increased capacity for conditioned medium from macrophage/fibroblast cocultures to degrade cartilage. This degradation could be inhibited by neutralising antibodies to IL- 6 and TNF- $\alpha{ }^{25}$

The implications of our in vitro study are that early events in the periprosthetic environment such as macrophage accu- 
mulation due to generation of particulate debris may have a substantial influence on tissue homeostasis. Our data suggest that macrophage recruitment and accumulation in response to wear particles create an interaction that is linked to the number of invading cells. While this constitutes a primary mechanism, the activation of the immune system, a direct interaction between macrophages and fibroblasts is less well established. We propose that an initiating event in particleinduced inflammation of the periprosthetic membrane is the initial influx of monocytes/macrophage to sites of particle accumulation. Our data show that although interactions between macrophages and fibroblasts can occur without activation of macrophages by wear particles, the presence of particulate debris exacerbates cytokine release.

This study was supported by the Orthopaedic Research Fund at Stanford University.

No benefits in any form have been received or will be received from a commercial party related directly or indirectly to the subject of this article.

\section{References}

1. Maloney WJ, Smith RL. Periprosthetic osteolysis in total hip arthroplasty: the role of particulate wear debris. Instr Course Lect 1996; 45:171-82.

2. Goodman SB. The cellular processes of aseptic loosening of joint arthroplasties. Seminars in Arthroplasty 1993;4:205-14.

3. Kwong LM, Jasty M, Mulroy RD, et al. The histology of the radiolucent line. J Bone Joint Surg [Br] 1992;74-B:67-73.

4. Willert HG. Reactions of the articular capsule to wear products of artificial joint prostheses. J Biomed Mater Res 1977;11:157-64.

5. Glant TT, Jacobs JJ. Response of three murine macrophage populations to particulate debris: bone resorption in organ cultures. J Orthop Res 1994;12:720-31.

6. Onodera S, Suzuki K, Matsuno T, Kaneda K. Macrophage migration inhibitory factor: its presence and speculated role in the tissues surrounding hip prosthesis. Trans Orthop Res Soc 1997;161.

7. Shanbhag AS, Jacobs JJ, Black J, Galante JO, Glant TT. Macrophage/particle interactions: effect of size, composition and surface area. J Biomed Mater Res 1994;28:81-9.

8. Horowitz SM, Gonzales JB. Effects of polyethylene on macrophages. J Orthop Res 1997;15:50-6.

9. Lee SH, Brennan FR, Jacobs JJ, et al. Human monocyte/macrophage response to cobalt-chromium corrosion products and titanium particles in patients with total joint replacements. J Orthop Res 1997;15:40-9.
10. Shanbhag AS, Jacobs JJ, Black J, Galante JO, Glant TT. Human monocyte response to particulate biomaterials generated in vivo and in vitro. J Orthop Res 1995;13:792-801.

11. Nakashima Y, Sun DH, Maloney WJ, et al. The expression of monocyte chemoattractant protein-1 (MCP-1) in particle-activated macrophages in vitro. Trans Orthop Res Soc 1997;22:24.

12. Nakashima Y, Chun L, Sun DH, et al. The expression of C-C chemokines in particle-activated macrophages in vivo and in vitro. Trans Orthop Res Soc 1998;23:790.

13. Schmalzried TP, Maloney WJ, Jasty M, Kwong LM, Harris WH. Autopsy studies of the bone-cement interface in well-fixed cemented total hip arthroplasties. J Arthroplasty 1993;8:179-88.

14. Maloney WJ, Smith RL, Castro F, Schurman DJ. Fibroblast response to metallic debris in vitro: enzyme induction, cell proliferation, and toxicity. J Bone Joint Surg [Am] 1993;75-A:835-44.

15. Frondoza CG, Tanner KT, Jones LC, Hungerford DS. Polymethylmethacrylate particles enhance DNA and protein synthesis of human fibroblasts in vitro. J Biomed Mater Res 1993;27:611-7.

16. Manlapaz M, Maloney WJH, Smith RL. In vitro activation of human fibroblasts by retrieved titanium alloy wear debris. $J$ Orthop Res 1996;14:465-72.

17. Shanbhag AS, Jacobs JJ, Black J, Galante JO, Glant TT. Effects of particles on fibroblast proliferation and bone resorption in vitro. Clin Orthop 1997;342:205-17.

18. Harigai M, Hara M, Kitani A, et al. Interleukin 1 and tumor necrosis factor-alpha synergistically increase the production of interleukin 6 in human synovial fibroblast. J Clin Lab Immunol 1991;34:107-13.

19. Calandra T, Bernhagen J, Mitchell RA, Bucala R. The macrophage is an important and previously unrecognized source of macrophage migration inhibitory factor. $J$ Exp Med 1994;179:1895-902.

20. Scamurra R, Arriaga C, Sprunger L, Baarsch MJ, Murtaugh MP. Regulation of interleukin-6 expression in porcine immune cells. J Interferon Cytokine Res 1997;16:289-96.

21. Lindroos PM, Coin PG, Badgett A, Morgan DL, Bonner JC. Alveolar macrophages stimulated with titanium dioxide, chrysotile asbestos, and residual oil fly ash upregulate the PDGF-receptor alpha on lung fibroblasts through an IL-1beta-dependent mechanism. Am J Respir Cell Mol Biol 1997;16:283-92.

22. Greenfield EM, Shaw SM, Gornik SA, Banks MA. Adenyl cyclase and interleukin 6 are downstream effectors of parathyroid hormone resulting in stimulation of bone resorption. J Clin Invest 1995;96: $1238-44$.

23. Manolagas SC. Role of cytokines in bone resorption. Bone 1995;17(2 Suppl):63-7.

24. Blue ML, Conrad P, Webb DL, Sarr T, Macaro M. Interacting monocytes and synoviocytes induce adhesion molecules by a cytokine-regulated process. Lymphokine Cytokine Res 1993;12:213-8.

25. Scott BB, Weisbrot LM, Greenwood J, et al. Rheumatoid arthritis synovial fibroblast and U937 macrophage/monocyte cell line interaction in cartilage degradation. Arthritis Rheum 1997;40:490-8. 